Acta Crystallographica Section E

Structure Reports

Online

ISSN 1600-5368

\section{Aminoguanidinium hydrogen fumarate}

\section{Swaminathan Murugavel, ${ }^{a}$ Gnanavelu Ganesh, ${ }^{\text {b }}$ Arunachalathevar Subbiah Pandi, ${ }^{{ } *}$ Subbiah Govindarajan $^{\mathrm{d}}$ and Rajendran Selvakumar ${ }^{\mathrm{d}}$}

a Department of Physics, Thanthai Periyar Government Institute of Technology, Vellore 632 002, India, 'b Department of Physics, SMK Fomra Institute of Technology, Thaiyur, Chennai 603 103, India, ' Department of Physics, Presidency College (Autonomous), Chennai 600 005, India, and ${ }^{\mathbf{d}}$ Department of Chemistry, Bharathiar University, Coimbatore 641 046, India

Correspondence e-mail: a_spandian@yahoo.com

Received 4 February 2009; accepted 8 February 2009

Key indicators: single-crystal X-ray study; $T=293 \mathrm{~K}$; mean $\sigma(\mathrm{C}-\mathrm{C})=0.002 \AA$; $R$ factor $=0.040 ; w R$ factor $=0.132 ;$ data-to-parameter ratio $=16.0$.

The title compound, $\mathrm{CH}_{7} \mathrm{~N}_{4}{ }^{+} \cdot \mathrm{C}_{4} \mathrm{H}_{3} \mathrm{O}_{4}{ }^{-}$, is a molecular salt in which the aminoguanidinium cations and fumarate monoanions are close to planar, with maximum deviations of 0.011 (1) and 0.177 (1) $\AA$, respectively. The crystal packing is stabilized by intermolecular $\mathrm{N}-\mathrm{H} \cdots \mathrm{O}$ and $\mathrm{O}-\mathrm{H} \cdots \mathrm{O}$ hydrogen bonds.

\section{Related literature}

For related structures, see: Adams (1977); Akella \& Keszler (1994); Mullen \& Hellner (1978). For biological applications, see: Makita et al. (1995); Brownlee et al. (1986).<smiles>N=C(N)NN</smiles>

\section{Experimental}

Crystal data

$\mathrm{CH}_{7} \mathrm{~N}_{4}{ }^{+} \cdot \mathrm{C}_{4} \mathrm{H}_{3} \mathrm{O}_{4}{ }^{-}$

$M_{r}=190.17$

Monoclinic, $P 2_{1} / c$ $\beta=114.688(3)^{\circ}$
$V=812.84(8) \AA^{3}$
$Z=4$
Mo $K \alpha$ radiation

$\mu=0.13 \mathrm{~mm}^{-1}$

$T=293 \mathrm{~K}$

$0.26 \times 0.15 \times 0.15 \mathrm{~mm}$

\section{Data collection}

Bruker APEXII CCD area-detector diffractometer

Absorption correction: multi-scan (SADABS; Sheldrick, 1996)

$T_{\min }=0.966, T_{\max }=0.976$

Refinement

$R\left[F^{2}>2 \sigma\left(F^{2}\right)\right]=0.040$

$w R\left(F^{2}\right)=0.132$

$S=1.04$

2340 reflections

146 parameters

10713 measured reflections 2340 independent reflections 1824 reflections with $I>2 \sigma(I)$ $R_{\text {int }}=0.028$

$\mathrm{H}$ atoms treated by a mixture of independent and constrained refinement

$\Delta \rho_{\max }=0.33{\mathrm{e} \AA^{-3}}^{-3}$

$\Delta \rho_{\min }=-0.31$ e $\AA^{-3}$

Table 1

Hydrogen-bond geometry $\left(\AA,^{\circ}\right)$.

\begin{tabular}{lllll}
\hline$D-\mathrm{H} \cdots A$ & $D-\mathrm{H}$ & $\mathrm{H} \cdots A$ & $D \cdots A$ & $D-\mathrm{H} \cdots A$ \\
\hline $\mathrm{O} 8-\mathrm{H} 8 \cdots \mathrm{O}^{\mathrm{i}}$ & 0.82 & 1.68 & $2.489(1)$ & 168 \\
$\mathrm{~N} 10-\mathrm{H} 10 A \cdots \mathrm{O}^{\mathrm{ii}}$ & $0.91(2)$ & $2.09(2)$ & $2.993(1)$ & $177(2)$ \\
$\mathrm{N} 11-\mathrm{H} 11 A \cdots \mathrm{O}^{\mathrm{ii}}$ & $0.92(2)$ & $1.91(2)$ & $2.827(2)$ & $173(2)$ \\
\hline
\end{tabular}

Symmetry codes: (i) $x-1, y, z$; (ii) $x-1,-y+\frac{3}{2}, z-\frac{1}{2}$.

Data collection: APEX2 (Bruker, 2004); cell refinement: APEX2; data reduction: $S A I N T$ (Bruker, 2004); program(s) used to solve structure: SHELXS97 (Sheldrick, 2008); program(s) used to refine structure: SHELXL97 (Sheldrick, 2008); molecular graphics: ORTEP-3 (Farrugia, 1997); software used to prepare material for publication: SHELXL97 and PLATON (Spek, 2009).

SM and ASP thank Dr Babu Vargheese, SAIF, IIT, Madras, India, for his help with the data collection.

Supplementary data and figures for this paper are available from the IUCr electronic archives (Reference: WM2220).

\title{
References
}

Adams, J. M. (1977). Acta Cryst. B33, 1513-1515.

Akella, A. \& Keszler, D. A. (1994). Acta Cryst. C50, 1974-1976.

Brownlee, M., Vlassara, H. \& Cerami, A. (1986). Diabetic Complications and Scientific and Clinical Aspects. London: Pitman.

Bruker (2004). APEX2, SAINT and XPREP. Bruker AXS Inc., Madison, Wisconsin, USA.

Farrugia, L. J. (1997). J. Appl. Cryst. 30, 565.

Makita, Z., Yanagisawa, K. \& Kuwajima, S. (1995). J. Diabetes Complications, 9, 265-268.

Mullen, D. \& Hellner, E. (1978). Acta Cryst. B34, 2789-2794.

Sheldrick, G. M. (1996). SADABS. University of Göttingen, Germany.

Sheldrick, G. M. (2008). Acta Cryst. A64, 112-122.

Spek, A. L. (2009). Acta Cryst. D65, 148-155. 


\section{supporting information}

Acta Cryst. (2009). E65, o548 [doi:10.1107/S1600536809004553]

\section{Aminoguanidinium hydrogen fumarate}

\section{Swaminathan Murugavel, Gnanavelu Ganesh, Arunachalathevar Subbiah Pandi, Subbiah Govindarajan and Rajendran Selvakumar}

\section{S1. Comment}

Aminoguanadine is an early inhibitor of advanced glycosylation end products (Makita et al., 1995). It helps prevent proteins cross-linking and is being used in diabetes, atherosclerosis, renal and aging disorders (Brownlee et al., 1986). Aminoguanadine is a highly reactive nucleophillic reagent that reacts with many biological molecules (pyridoxal phosphate, pyruvate, glucose, malondialdehyde, and others). The crystal structures of several guanidinium salts have previously been reported over the last three decades (Adams, 1977; Mullen \& Hellner, 1978). Here we report the crystal structure of the title compound, aminoguanidinium hydrogenfumarate, (I), (Fig. 1). In the molecular salt (I), the aminoguanidinium cation and fumarate anion each are nearly planar, with maximum deviations of -0.011 (1) $\AA$ and -0.177 (1) $\AA$ for atom N12 and O7, respectively ( Fig. 1). The bond lengths in (I) are comparable with the corresponding values observed in related structures (Akella \& Keszler , 1994). The angle between the best planes of the aminoguanidinium cation and the fumarate anion is $12.78(6)^{\circ}$. Atom N10 and N11 in the molecule at $(x, y, z)$ donate one proton each to the atoms $\mathrm{O} 7$ and $\mathrm{O} 6$ in the molecule at $(-1+x, 3 / 2-y,-1 / 2+z)$, generating a $R_{2}{ }^{2}(8)$ ring motif (Table 1 and Fig. 2). Also, an $\mathrm{O}$ $-\mathrm{H} \cdots \mathrm{O}$ interaction is observed (Table 1 ). Thus, the symmetry-related molecules are cross linked by these hydrogen bonds to generate a three-dimensional network.

\section{S2. Experimental}

Needle-shaped single crystals of aminoguanidium hydrogenfumarate were prepared by slow evaporation of the aqueous solution obtained by dissolving of aminoguanidinium hydrogencarbonate $(0.136 \mathrm{~g} ; 0.001 \mathrm{~mol})$ in fumaric acid $(0.116 \mathrm{~g} ; 1$ mmol) solution $(30 \mathrm{~mL})$ at ambient condition. Colourless single crystals suitable for X-ray diffraction obtained after four days were collected, washed with ethanol and air dried.

\section{S3. Refinement}

All $\mathrm{N}$ bound $\mathrm{H}$ atoms were located in a difference map and refined freely. All other $\mathrm{H}$ atoms were fixed geometrically and allowed to ride on their parent atoms, with distances of $\mathrm{O}-\mathrm{H}=0.82 \AA$ and $\mathrm{C}-\mathrm{H}=0.93 \AA$ with $U_{\text {iso }}(\mathrm{H})=1.2 U_{\text {eq. }}$. 


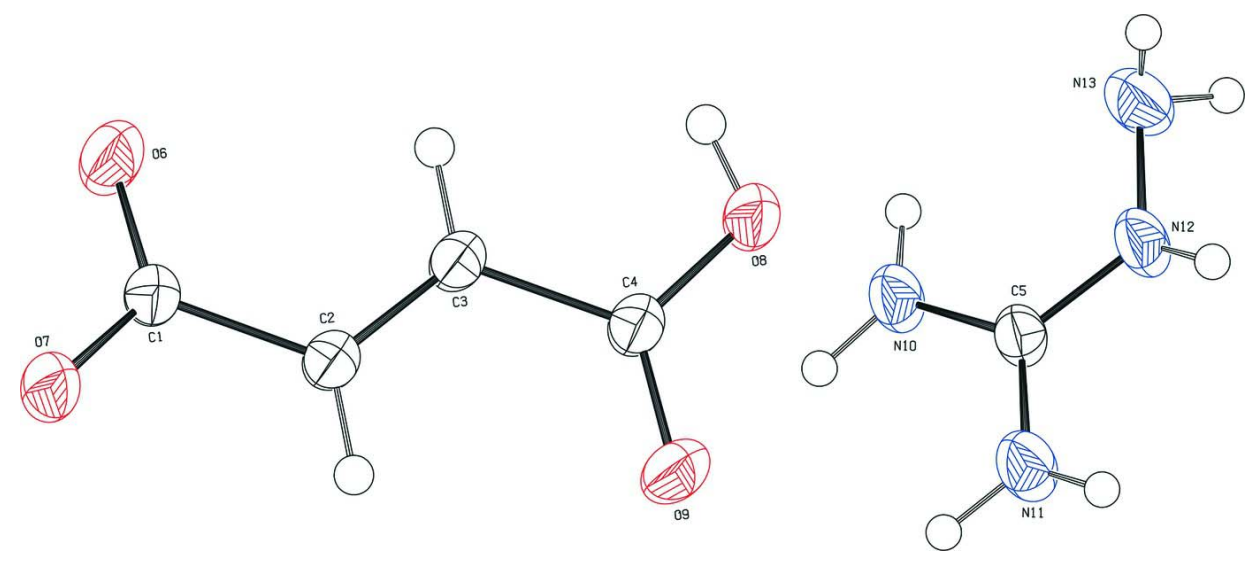

\section{Figure 1}

The molecular structure of the ions present in compound (I) showing 50\% probability displacement ellipsoids.

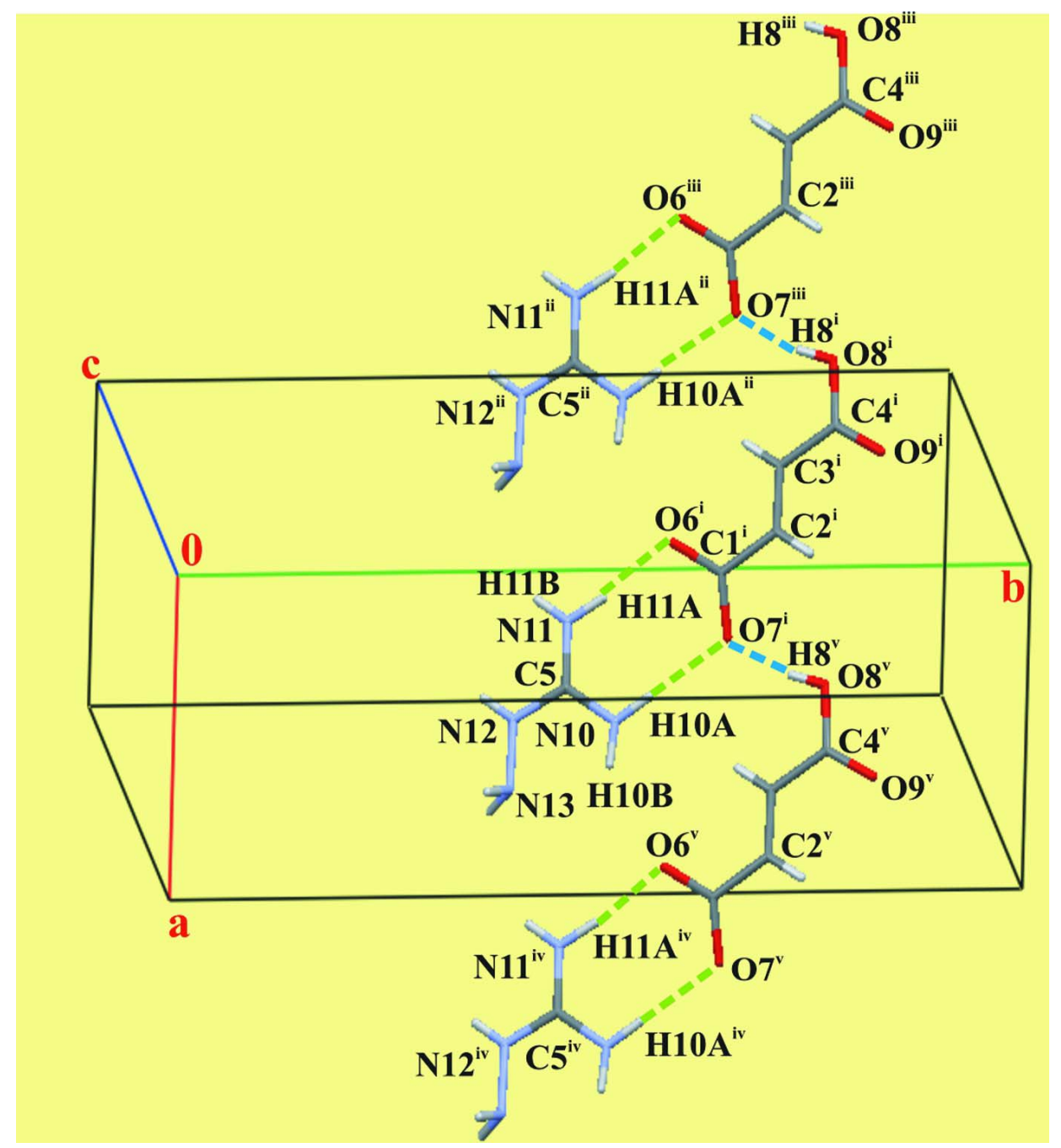

\section{Figure 2}

$\mathrm{N}-\mathrm{H} \cdots \mathrm{O}$ and $\mathrm{O}-\mathrm{H} \cdots \mathrm{O}$ hydrogen bonds (dotted lines) in the title compound. [Symmetry codes: (i) $\mathrm{x}-1,-\mathrm{y}+3 / 2, \mathrm{z}-1 / 2$; (ii) $\mathrm{x}-1, \mathrm{y}, \mathrm{z}]$. 


\section{Aminoguanidinium hydrogen fumarate}

Crystal data

$\mathrm{CH}_{7} \mathrm{~N}_{4}^{+} \cdot \mathrm{C}_{4} \mathrm{H}_{3} \mathrm{O}_{4}^{-}$

$M_{r}=190.17$

Monoclinic, $P 2_{1} / c$

Hall symbol: -P $2 \mathrm{ybc}$

$a=6.3869$ (3) $\AA$

$b=19.8731(10) \AA$

$c=7.0482(4) \AA$

$\beta=114.688(3)^{\circ}$

$V=812.84(8) \AA^{3}$

$Z=4$

$F(000)=400$

$D_{\mathrm{x}}=1.554 \mathrm{Mg} \mathrm{m}^{-3}$

Mo $K \alpha$ radiation, $\lambda=0.71073 \AA$

Cell parameters from 1824 reflections

$\theta=2-29.9^{\circ}$

$\mu=0.13 \mathrm{~mm}^{-1}$

$T=293 \mathrm{~K}$

Block, colourless

$0.26 \times 0.15 \times 0.15 \mathrm{~mm}$

\section{Data collection}

Bruker APEXII CCD area-detector diffractometer

Radiation source: fine-focus sealed tube

Graphite monochromator

Detector resolution: 10.0 pixels $\mathrm{mm}^{-1}$

$\omega$ scans

Absorption correction: multi-scan

(SADABS; Sheldrick, 1996)

$T_{\min }=0.966, T_{\max }=0.976$

Refinement

Refinement on $F^{2}$

Least-squares matrix: full

$R\left[F^{2}>2 \sigma\left(F^{2}\right)\right]=0.040$

$w R\left(F^{2}\right)=0.132$

$S=1.04$

2340 reflections

146 parameters

0 restraints

Primary atom site location: structure-invariant direct methods

10713 measured reflections

2340 independent reflections

1824 reflections with $I>2 \sigma(I)$

$R_{\text {int }}=0.028$

$\theta_{\max }=29.9^{\circ}, \theta_{\min }=2.1^{\circ}$

$h=-8 \rightarrow 7$

$k=-24 \rightarrow 27$

$l=-9 \rightarrow 9$
Secondary atom site location: difference Fourier map

Hydrogen site location: inferred from neighbouring sites

$\mathrm{H}$ atoms treated by a mixture of independent and constrained refinement

$w=1 /\left[\sigma^{2}\left(F_{\mathrm{o}}^{2}\right)+(0.0756 P)^{2}+0.1408 P\right]$ where $P=\left(F_{\mathrm{o}}^{2}+2 F_{\mathrm{c}}{ }^{2}\right) / 3$

$(\Delta / \sigma)_{\max }<0.001$

$\Delta \rho_{\max }=0.33$ e $\AA^{-3}$

$\Delta \rho_{\min }=-0.31 \mathrm{e} \AA^{-3}$

\section{Special details}

Geometry. All esds (except the esd in the dihedral angle between two 1.s. planes) are estimated using the full covariance matrix. The cell esds are taken into account individually in the estimation of esds in distances, angles and torsion angles; correlations between esds in cell parameters are only used when they are defined by crystal symmetry. An approximate (isotropic) treatment of cell esds is used for estimating esds involving l.s. planes.

Refinement. Refinement of $\mathrm{F}^{2}$ against ALL reflections. The weighted R-factor wR and goodness of fit $\mathrm{S}$ are based on $\mathrm{F}^{2}$, conventional R-factors $R$ are based on $F$, with $F$ set to zero for negative $F^{2}$. The threshold expression of $F^{2}>2 \operatorname{sigma}\left(F^{2}\right)$ is used only for calculating R-factors(gt) etc. and is not relevant to the choice of reflections for refinement. R-factors based on $\mathrm{F}^{2}$ are statistically about twice as large as those based on F, and R-factors based on ALL data will be even larger.

Fractional atomic coordinates and isotropic or equivalent isotropic displacement parameters $\left(\AA^{2}\right)$

\begin{tabular}{lllll}
\hline & $x$ & $y$ & $z$ & $U_{\text {iso }} * U_{\text {eq }}$ \\
\hline H11B & $0.243(3)$ & $0.4484(12)$ & $0.254(3)$ & $0.057(5)^{*}$ \\
H12 & $0.573(3)$ & $0.3932(11)$ & $0.302(3)$ & $0.056(5)^{*}$ \\
H13A & $0.861(4)$ & $0.3941(12)$ & $0.204(3)$ & $0.072(6)^{*}$
\end{tabular}




$\begin{array}{lllll}\text { C1 } & 1.12336(19) & 0.84396(5) & 0.68336(19) & 0.0281(3) \\ \text { C2 } & 0.9984(2) & 0.78049(5) & 0.68320(19) & 0.0274(2) \\ \text { H2 } & 1.0738 & 0.7396 & 0.6953 & 0.033^{*} \\ \text { C3 } & 0.7863(2) & 0.78034(6) & 0.6666(2) & 0.0292(3) \\ \text { H3 } & 0.7125 & 0.8212 & 0.6595 & 0.035^{*} \\ \text { C4 } & 0.6603(2) & 0.71704(5) & 0.65888(19) & 0.0279(3) \\ \text { O6 } & 1.04931(17) & 0.89803(5) & 0.71551(19) & 0.0466(3) \\ \text { O7 } & 1.30209(15) & 0.83829(4) & 0.64654(17) & 0.0362(2) \\ \text { O8 } & 0.45191(15) & 0.72255(4) & 0.64769(17) & 0.0374(2) \\ \text { H8 } & 0.4189 & 0.7625 & 0.6465 & 0.056^{*} \\ \text { O9 } & 0.74557(17) & 0.66199(4) & 0.66416(19) & 0.0434(3) \\ \text { H10A } & 0.521(3) & 0.5783(9) & 0.214(3) & 0.046(5)^{*} \\ \text { H10B } & 0.747(4) & 0.5370(10) & 0.245(3) & 0.057(5)^{*} \\ \text { H11A } & 0.221(3) & 0.5225(10) & 0.224(3) & 0.053(5)^{*} \\ \text { C5 } & 0.5133(2) & 0.48405(6) & 0.25398(18) & 0.0277(3) \\ \text { N10 } & 0.6094(2) & 0.54110(5) & 0.23653(19) & 0.0350(3) \\ \text { N11 } & 0.3028(2) & 0.48299(6) & 0.2428(2) & 0.0412(3) \\ \text { N12 } & 0.6265(2) & 0.42625(5) & 0.2812(2) & 0.0361(3) \\ \text { N13 } & 0.8519(2) & 0.42556(6) & 0.2974(2) & 0.0431(3) \\ \text { H13B } & 0.935(4) & 0.4125(10) & 0.424(3) & 0.061(6)^{*} \\ & & & \end{array}$

Atomic displacement parameters $\left(\AA^{2}\right)$

\begin{tabular}{lllllll}
\hline & $U^{11}$ & $U^{22}$ & $U^{33}$ & $U^{12}$ & $U^{13}$ & $U^{23}$ \\
\hline C1 & $0.0233(5)$ & $0.0217(5)$ & $0.0413(6)$ & $-0.0015(4)$ & $0.0153(5)$ & $0.0003(4)$ \\
C2 & $0.0253(5)$ & $0.0198(5)$ & $0.0400(6)$ & $-0.0004(4)$ & $0.0166(5)$ & $0.0010(4)$ \\
C3 & $0.0270(6)$ & $0.0179(5)$ & $0.0477(7)$ & $-0.0010(4)$ & $0.0206(5)$ & $-0.0004(4)$ \\
C4 & $0.0264(5)$ & $0.0200(5)$ & $0.0412(6)$ & $-0.0018(4)$ & $0.0180(5)$ & $0.0000(4)$ \\
O6 & $0.0395(5)$ & $0.0226(4)$ & $0.0891(8)$ & $-0.0035(4)$ & $0.0382(6)$ & $-0.0077(5)$ \\
O7 & $0.0291(4)$ & $0.0264(4)$ & $0.0620(6)$ & $-0.0027(3)$ & $0.0279(4)$ & $0.0003(4)$ \\
O8 & $0.0280(4)$ & $0.0228(4)$ & $0.0683(7)$ & $-0.0027(3)$ & $0.0272(4)$ & $-0.0004(4)$ \\
O9 & $0.0390(5)$ & $0.0196(4)$ & $0.0796(7)$ & $0.0023(3)$ & $0.0328(5)$ & $0.0019(4)$ \\
C5 & $0.0301(6)$ & $0.0208(5)$ & $0.0345(6)$ & $0.0007(4)$ & $0.0158(5)$ & $0.0002(4)$ \\
N10 & $0.0341(6)$ & $0.0206(5)$ & $0.0553(7)$ & $-0.0007(4)$ & $0.0238(5)$ & $0.0025(4)$ \\
N11 & $0.0350(6)$ & $0.0254(5)$ & $0.0713(9)$ & $0.0000(5)$ & $0.0304(6)$ & $0.0024(5)$ \\
N12 & $0.0353(6)$ & $0.0193(5)$ & $0.0599(7)$ & $0.0021(4)$ & $0.0260(5)$ & $0.0052(4)$ \\
N13 & $0.0345(6)$ & $0.0348(6)$ & $0.0638(9)$ & $0.0076(5)$ & $0.0244(6)$ & $0.0025(6)$ \\
& & & & & & \\
\hline
\end{tabular}

Geometric parameters $\left(A,{ }^{\circ}\right)$

\begin{tabular}{llll}
\hline $\mathrm{C} 1-\mathrm{O} 6$ & $1.2325(14)$ & $\mathrm{C} 5-\mathrm{N} 10$ & $1.3190(15)$ \\
$\mathrm{C} 1-\mathrm{O} 7$ & $1.2770(14)$ & $\mathrm{C} 5-\mathrm{N} 12$ & $1.3278(15)$ \\
$\mathrm{C} 1-\mathrm{C} 2$ & $1.4922(15)$ & $\mathrm{N} 10-\mathrm{H} 10 \mathrm{~A}$ & $0.905(18)$ \\
$\mathrm{C} 2-\mathrm{C} 3$ & $1.3105(16)$ & $\mathrm{N} 10-\mathrm{H} 10 \mathrm{~B}$ & $0.86(2)$ \\
$\mathrm{C} 2-\mathrm{H} 2$ & 0.9300 & $\mathrm{~N} 11-\mathrm{H} 11 \mathrm{~B}$ & $0.80(2)$ \\
$\mathrm{C} 3-\mathrm{C} 4$ & $1.4820(15)$ & $\mathrm{N} 11-\mathrm{H} 11 \mathrm{~A}$ & $0.92(2)$ \\
$\mathrm{C} 3-\mathrm{H} 3$ & 0.9300 & $\mathrm{~N} 12-\mathrm{N} 13$ & $1.3960(16)$ \\
$\mathrm{C} 4-\mathrm{O} 9$ & $1.2159(14)$ & $\mathrm{N} 12-\mathrm{H} 12$ & $0.78(2)$
\end{tabular}




\begin{tabular}{llll}
$\mathrm{C} 4-\mathrm{O} 8$ & $1.3044(14)$ & $\mathrm{N} 13-\mathrm{H} 13 \mathrm{~A}$ & $0.93(2)$ \\
$\mathrm{O} 8-\mathrm{H} 8$ & 0.8200 & $\mathrm{~N} 13-\mathrm{H} 13 \mathrm{~B}$ & $0.87(2)$ \\
$\mathrm{C} 5-\mathrm{N} 11$ & $1.3136(17)$ & & \\
& & & \\
$\mathrm{O} 6-\mathrm{C} 1-\mathrm{O} 7$ & $123.96(10)$ & $\mathrm{N} 11-\mathrm{C} 5-\mathrm{N} 12$ & $118.46(11)$ \\
$\mathrm{O} 6-\mathrm{C} 1-\mathrm{C} 2$ & $119.40(10)$ & $\mathrm{N} 10-\mathrm{C} 5-\mathrm{N} 12$ & $120.71(11)$ \\
$\mathrm{O} 7-\mathrm{C} 1-\mathrm{C} 2$ & $116.64(10)$ & $\mathrm{C} 5-\mathrm{N} 10-\mathrm{H} 10 \mathrm{~A}$ & $115.9(12)$ \\
$\mathrm{C} 3-\mathrm{C} 2-\mathrm{C} 1$ & $122.32(10)$ & $\mathrm{C} 5-\mathrm{N} 10-\mathrm{H} 10 \mathrm{~B}$ & $129.6(14)$ \\
$\mathrm{C} 3-\mathrm{C} 2-\mathrm{H} 2$ & 118.8 & $\mathrm{H} 10 \mathrm{~A}-\mathrm{N} 10-\mathrm{H} 10 \mathrm{~B}$ & $121.4(15)$ \\
$\mathrm{C} 1-\mathrm{C} 2-\mathrm{H} 2$ & 118.8 & $\mathrm{C} 5-\mathrm{N} 11-\mathrm{H} 11 \mathrm{~B}$ & $120.0(12)$ \\
$\mathrm{C} 2-\mathrm{C} 3-\mathrm{C} 4$ & $122.04(10)$ & $\mathrm{C} 5-\mathrm{N} 11-\mathrm{H} 11 \mathrm{~A}$ & $119(2)$ \\
$\mathrm{C} 2-\mathrm{C} 3-\mathrm{H} 3$ & 119.0 & $\mathrm{H} 11 \mathrm{~B}-\mathrm{N} 11-\mathrm{H} 11 \mathrm{~A}$ & $120.06(11)$ \\
$\mathrm{C} 4-\mathrm{C} 3-\mathrm{H} 3$ & 119.0 & $\mathrm{C} 5-\mathrm{N} 12-\mathrm{N} 13$ & $120.1(15)$ \\
$\mathrm{O} 9-\mathrm{C} 4-\mathrm{O} 8$ & $120.67(10)$ & $\mathrm{C} 5-\mathrm{N} 12-\mathrm{H} 12$ & $119.5(15)$ \\
$\mathrm{O} 9-\mathrm{C} 4-\mathrm{C} 3$ & $122.22(10)$ & $\mathrm{N} 13-\mathrm{N} 12-\mathrm{H} 12$ & $108.3(15)$ \\
$\mathrm{O} 8-\mathrm{C} 4-\mathrm{C} 3$ & $117.11(10)$ & $\mathrm{N} 12-\mathrm{N} 13-\mathrm{H} 13 \mathrm{~A}$ & $104.9(14)$ \\
$\mathrm{C} 4-\mathrm{O} 8-\mathrm{H} 8$ & 109.5 & $\mathrm{~N} 12-\mathrm{N} 13-\mathrm{H} 13 \mathrm{~B}$ & $109.7(19)$ \\
$\mathrm{N} 11-\mathrm{C} 5-\mathrm{N} 10$ & $120.83(11)$ & $\mathrm{H} 13 \mathrm{~A}-\mathrm{N} 13-\mathrm{H} 13 \mathrm{~B}$ & $178.28(11)$ \\
& & & $-178.90(13)$ \\
$\mathrm{O} 6-\mathrm{C} 1-\mathrm{C} 2-\mathrm{C} 3$ & $16.50(19)$ & $\mathrm{C} 2-\mathrm{C} 3-\mathrm{C} 4-\mathrm{O} 8$ & $1.8(2)$ \\
$\mathrm{O} 7-\mathrm{C} 1-\mathrm{C} 2-\mathrm{C} 3$ & $-162.59(12)$ & $\mathrm{N} 11-\mathrm{C} 5-\mathrm{N} 12-\mathrm{N} 13$ & \\
$\mathrm{C} 1-\mathrm{C} 2-\mathrm{C} 3-\mathrm{C} 4$ & $177.74(11)$ & $\mathrm{N} 10-\mathrm{C} 5-\mathrm{N} 12-\mathrm{N} 13$ & \\
$\mathrm{C} 2-\mathrm{C} 3-\mathrm{C} 4-\mathrm{O} 9$ & $-1.2(2)$ & & \\
\hline
\end{tabular}

Hydrogen-bond geometry $\left(\AA,{ }^{\circ}\right)$

\begin{tabular}{lllll}
\hline$D-\mathrm{H} \cdots A$ & $D-\mathrm{H}$ & $\mathrm{H} \cdots A$ & $D \cdots A$ & $D-\mathrm{H} \cdots A$ \\
\hline $\mathrm{O} 8-\mathrm{H} 8 \cdots \mathrm{O} 7^{\mathrm{i}}$ & 0.82 & 1.68 & $2.489(1)$ & 168 \\
$\mathrm{~N} 10-\mathrm{H} 10 A \cdots \mathrm{O} 7^{\mathrm{ii}}$ & $0.91(2)$ & $2.09(2)$ & $2.993(1)$ & $177(2)$ \\
$\mathrm{N} 11-\mathrm{H} 11 A \cdots{ }^{\mathrm{ii}}$ & $0.92(2)$ & $1.91(2)$ & $2.827(2)$ & $173(2)$ \\
\hline
\end{tabular}

Symmetry codes: (i) $x-1, y, z$; (ii) $x-1,-y+3 / 2, z-1 / 2$. 